\title{
CP VIOLATION IN THE STANDARD MODEL AND BEYOND
}

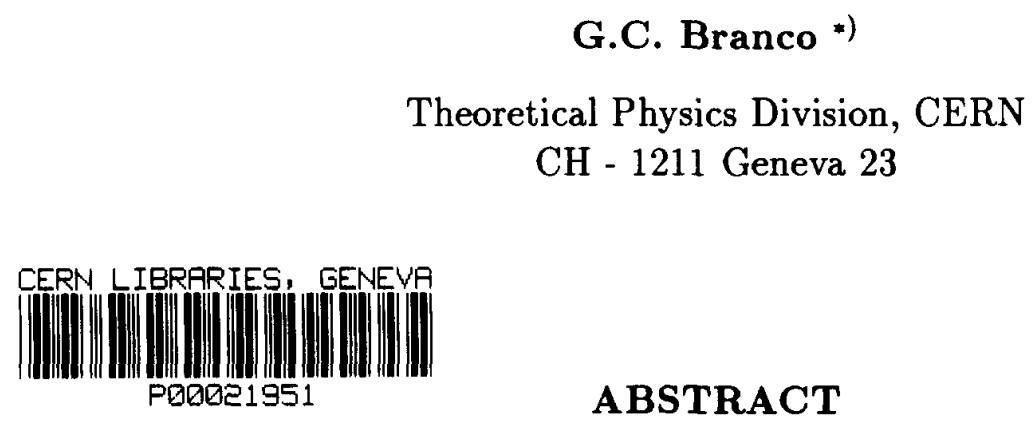

We present an overview of $\mathrm{CP}$ violation in the Standard Model and some of its minimal extensions.

Lectures given at

Mexican School on Particle Physics

Guanajuato, Mexico, December 1992

*) Permanent address: Departamento de Fisica and CFIF/UTL, Instituto Superior Técnico, Avenida Rovisco Pais, 1096 Lisboa Codex, Portugal.

CERN-TH.7176/94

February 1994 


\section{Introduction}

Understanding the origin of CP violation is one of the outstanding open questions in particle physics [1]. Although one can incorporate CP violation in the three-generations Standard Model (SM) through the Kobayashi-Maskawa (KM) mechanism [2], there is no deep understanding of the origin of $\mathrm{CP}$ violation. From the experimental point of view, very little is also known. At present, CP violation has only been observed in the $K^{0}-\bar{K}^{0}$ sector and indeed all the experimental observations are consistent with the original superweak model [3] suggested by Wolfenstein soon after the discovery of the decay $K_{L} \rightarrow \pi^{+} \pi^{-}$, by Christensen, Cronin, Fitch and Turlay [4].

On the other hand, the present bounds on the electric dipole moment of the neutron and the electron imply significant restrictions on physics beyond the SM, while the observed baryon asymmetry may provide motivation for extending the SM [5]. Indeed the indication that the amount of CP violation one has in the SM through the KM mechanism is probably not sufficient to generate the observed baryon asymmetry [5] suggests other sources of CP violation beyond the SM.

\section{The Standard Model}

\subsection{The Kobayashi-Maskawa mechanism}

In the standard $S U(2) \times U(1)$ electroweak gauge theory with one Higgs doublet, the only way to introduce $\mathrm{CP}$ violation is by having complex Yukawa couplings:

$$
\mathcal{L}_{Y}=g_{d}\left(\bar{u}^{0} \bar{d}^{0}\right)_{L} \Phi d_{R}^{0}+g_{u}\left(\bar{u}^{0} \bar{d}^{0}\right)_{L} \tilde{\Phi} u_{R}^{0}+\text { h.c. }
$$

where $u^{0}, d^{0}$ stand for quark weak-eigenstates, $\Phi$ is the weak Higgs doublet, and $\tilde{\Phi}=i \sigma_{2} \Phi^{*}$. We have suppressed the family indices, so for $n$ families, $g_{d}, g_{u}$ will be $n \times n$ matrices in flavour space. The charged-current couplings are diagonal at this stage:

$$
\mathcal{L}_{W}=\frac{g}{\sqrt{2}}\left[\bar{u}_{L}^{0} \gamma_{\mu} d_{L}^{0} W^{\mu}+\text { h.c. }\right]
$$

Gauge symmetry breaking generates arbitrary complex quark mass matrices $M_{u}, M_{d}$ which can be diagonalized through the bi-unitary transformations:

$$
\begin{aligned}
u_{L}^{0} \rightarrow U_{L}^{u} u_{L} ; \quad d_{L}^{0}= & U_{L}^{d} d_{L} ; \quad u_{R}^{0} \rightarrow U_{R}^{u} u_{R} ; \quad d_{r}^{0}=U_{R}^{d} d_{R} \\
U_{L}^{u^{\dagger}} M_{u} U_{R}^{u} & =D_{u} \\
U_{L}^{d^{\dagger}} M_{d} U_{R}^{d} & =D_{d}
\end{aligned}
$$


where $u, d$ stand for the quark mass eigenstates and $D_{u}, D_{d}$ are the diagonal quark mass matrices. When expressed in terms of the mass eigenstates, the charged weak current in the quark sector is no longer diagonal and the interaction is given by:

$$
\mathcal{L}_{W}=\frac{g}{\sqrt{2}} \bar{u}_{L} \gamma^{\mu} V^{\mathrm{CKM}} d_{L} W_{\mu}+\text { h.c. }
$$

where $V^{\mathrm{CKM}}=U_{L}^{u^{\dagger}} U_{L}^{d}$. If one makes a redefinition of the quark field phases:

$$
u_{i} \rightarrow \exp \left(i \alpha_{i}\right) u_{i} ; \quad d_{j} \rightarrow \exp \left(i \beta_{j}\right) d_{j}
$$

the CKM matrix transforms as:

$$
V_{i j}^{\mathrm{CKM}} \rightarrow V_{i j}^{\mathrm{CKM}} \exp i\left(\beta_{j}-\alpha_{i}\right)
$$

By using the freedom to redefine the quark phases, one can remove $(2 n-1)$ phases from $V^{\mathrm{CKM}}$, since an overall change of all phases leaves $V^{\mathrm{CKM}}$ invariant. Since an $n$-dimensional unitary matrix has $n^{2}$ parameters, $1 / 2 n(n-1)$ of which are used to parametrize the $O(n)$ rotation, the number of physical phases in $V^{\mathrm{CKM}}$ is given by:

$$
N_{\phi}=n^{2}-\frac{1}{2} n(n-1)-(2 n-1)=\frac{1}{2}(n-1)(n-2) .
$$

From Eq. (7) one concludes that for three or more generations there will be (a) physical phase(s) which lead to CP violation. This is the crucial observation made by Kobayashi and Maskawa [2] in their seminal paper. It is clear that only functions of $V^{\mathrm{CKM}}$ which are rephasing invariant can be observable. The simplest invariants are the moduli $\left|V_{i j}\right|$ and the quartic products $V_{i j} V_{k \ell} V_{i \ell}^{*} V_{k j}^{*}$, which are usually designated "quartets". It can easily be shown that any higher order invariant $\left(V_{i j} V_{k \ell} V_{m n} \ldots V_{r s} V_{i \ell}^{*} V_{k n}^{*} \ldots V_{r j}^{*}\right)$ can be expressed in terms of moduli and quartets. For example, in the case of a sextet, one has:

$$
V_{i j} V_{k \ell} V_{m n} V_{i \ell}^{*} V_{k n}^{*} V_{m j}^{*}=\frac{\left(V_{i j} V_{k \ell} V_{i \ell}^{*} V_{k j}^{*}\right)\left(V_{k j} V_{m n} V_{k n}^{*} V_{m j}^{*}\right)}{\left|V_{k j}\right|^{2}}
$$

So far our discussion has been for an arbitrary number of generations. For three generations, the imaginary parts of all quartets have the same absolute value, as a result of unitarity. For example, orthogonality of the first two rows of $V^{\text {CKM }}$ implies

$$
\left(V_{11} V_{21}^{*}+V_{13} V_{23}^{*}\right) V_{12}^{*} V_{22}=-\left|V_{12}\right|^{2}\left|V_{22}\right|^{2}
$$

and taking the imaginary part of Eq. (9):

$$
\operatorname{Im}\left(V_{11} V_{22} V_{21}^{*} V_{12}^{*}\right)=-\operatorname{Im}\left(V_{13} V_{22} V_{12}^{*} V_{23}^{*}\right)
$$

The fact that in the three-generation SM the absolute value of the imaginary part of all quartets is the same, enables one to identify the strength of CP violation with the quantity $\delta_{\mathrm{KM}}$ defined by:

$$
\delta_{\mathrm{KM}}=\left|\operatorname{Im} V_{c d} V_{u b} V_{c b}^{*} V_{u d}^{*}\right|
$$


where $V_{u b} \equiv V_{13}$, etc. The smallness of $\delta_{\mathrm{KM}}$ just reflects the smallness of some of the CKM matrix elements. Indeed from Eq. (11) and taking into account the experimental values of $\left|V_{i j}\right|$, one obtains the bound

$$
\delta_{\mathrm{KM}} \leq 10^{-4}
$$

The strength of CP violation has an interesting geometrical interpretation. If one writes the unitarity relation:

$$
V_{u d} V_{u b}^{*}+V_{c d} V_{c b}^{*}+V_{t d} V_{t b}^{*}=0
$$

in the complex plane, it can be readily verified that

$$
\delta_{\mathrm{KM}}=\frac{1}{2} A
$$

where $A$ is the area of the triangle formed by the three complex quantities of Eq. (13). Needless to say, one can form other unitarity triangles, but in the $3 g \mathrm{SM}$, all unitarity triangles have the same area. In the $3 g \mathrm{SM}$, one can express $\delta_{\mathrm{KM}}$ in terms of four independent moduli [6]

$$
\delta_{\mathrm{KM}}=\left[U_{11} U_{22} U_{12} U_{21}-R^{2}\right]^{1 / 2}
$$

where $U_{i j} \equiv\left|V_{i j}\right|^{2}$ and $R$ is given by:

$$
R=\frac{1}{2}\left[1-U_{11}-U_{22}-U_{12}-U_{21}+U_{11} U_{22}+U_{12} U_{21}\right]
$$

This is a remarkable property of the $3 g \mathrm{SM}$, since it implies that if one could measure with sufficient precision four independent moduli (through CP conserving processes) one could get the strength of $\mathrm{CP}$ violation in the $3 g \mathrm{SM}$. At present, this is not possible, due to both the relative size of the various $\left|V_{i j}\right|$ and the relatively large experimental errors.

As a result of the rephasing freedom of Eq. (6), it is clear that there are many alternative ways to parametrize the CKM matrix. The most used ones are the so-called standard parametrization [7] and the Wolfenstein parametrization [8]. They are well described in the literature and we will not present them here. Instead we would like to refer to alternative ways of parametrizing the CKM matrix which only use directly measurable quantities which have to be rephasing invariant. We will present two examples of rephasing invariant parametrizations, one using three moduli and the argument of a quartet as input parameters, and another using four moduli as input parameters.

\section{Three moduli and one invariant phase}

This parametrization was proposed by Bjorken and Dunietz [9] who suggest the following basic parameters:

$$
\left|V_{u s}\right| ;\left|V_{u b}\right| ;\left|V_{c b}\right| ; \arg \left[V_{c b} V_{u s} V_{c s}^{*} V_{u b}^{*}\right]
$$

It is suggested that the reconstruction of the full matrix be done by choosing $V_{u d}, V_{u s}, V_{c b}, V_{t b}, V_{c s}$ real and positive. 


\section{Four moduli}

This parametrization [6] uses four moduli and a possible choice is:

$$
\left|V_{u s}\right| ;\left|V_{u b}\right| ;\left|V_{c b}\right| ;\left|V_{t d}\right|
$$

The magnitude of $\delta_{\mathrm{KM}}$ can be derived by using an expression analogous to the ones given in Eqs. (15), (16).

\subsection{Invariants and $\mathrm{CP}$ violation}

In this section we address the following question: given a Lagrangian, how can one verify whether it violates CP invariance? We will show next that the simplest way to study the CP properties of a Lagrangian is by investigating whether it is possible to construct a CP transformation which leaves the Lagrangian invariant. If the existence of such a transformation requires non-trivial restrictions on some of the couplings, then the Lagrangian can violate CP. We will illustrate the application of the method by considering first the SM and then indicating how it can be easily extended to models beyond the SM. The part of the SM Lagrangian which is relevant to the study of the CP properties is:

$$
\mathcal{L}=\mathcal{L}_{Y}+\mathcal{L}_{W}
$$

where $\mathcal{L}_{Y}, \mathcal{L}_{W}$ are given by Eqs. (1), (2). The most general CP transformation which leaves the gauge interactions invariant is:

$$
\begin{array}{lll}
u_{L} \rightarrow W_{L} C u_{L}^{*} & ; \quad u_{R} \rightarrow W_{R}^{u} C u_{R}^{*} \\
d_{L} \rightarrow W_{L} C d_{L}^{*} & ; \quad d_{R} \rightarrow W_{R}^{d} C d_{R}^{*}
\end{array}
$$

where $W_{L}, W_{R}^{u}, W_{R}^{d}$ are $n$-dimensional unitary matrices acting in flavour space. It is clear that the left-handed charged current interactions constrain $u_{L}, d_{L}$ to transform in the same way, while the absence of right-handed charged currents in the SM allows $u_{R}, d_{R}$ to transform differently under CP. In order for $\mathcal{L}_{Y}$ to be invariant under Eq. (20) the matrices $g_{d}, g_{u}$ are constrained to satisfy the conditions $[10]$

$$
\begin{aligned}
& W_{L}^{\dagger} g_{u} W_{R}^{u}=g_{u}^{*} \\
& W_{L}^{\dagger} g_{d} W_{R}^{d}=g_{d}^{*}
\end{aligned}
$$

From Eqs. (21) one obtains:

$$
\begin{aligned}
& W_{L}^{\dagger} G_{u} W_{L}=G_{u}^{*} \\
& W_{L}^{\dagger} G_{d} W_{L}=G_{d}^{*}
\end{aligned}
$$

where $G_{u}=g_{u} g_{u}^{\dagger}, G_{d}=g_{d} g_{d}^{\dagger}$. It can be shown [10] that if there is a matrix $W_{L}$ satisfying simultaneously Eqs. (22), (23), then there always exist matrices $W_{R}^{u}, W_{R}^{d}$ satisfying Eqs. (21). Therefore, a necessary and sufficient condition for the Lagrangian of the SM to be CP invariant 
is the existence of a matrix $W_{L}$ satisfying Eqs. (22), (23). From these equations one can now derive non-trivial CP restrictions on the Yukawa couplings, expressed in terms of weak-basis invariants. From Eqs. (22), (23), one obtains [10]

$$
W_{L}^{\dagger}\left[G_{u}^{p}, G_{d}^{q}\right] W_{L}=-\left[G_{u}^{p}, G_{d}^{q}\right]^{T} \quad p, q \text { integers }
$$

Note that the minus sign on the right-hand side of Eq. (24) is crucial. If one multiplies Eq. (24) by itself an odd number of times and takes traces, one derives:

$$
\operatorname{tr}\left[G_{u}^{p}, G_{d}^{q}\right]^{r}=0 \quad r \text { odd }
$$

Equations (25) are the necessary conditions to have CP invariance, expressed in terms of weak basis invariants and valid for an arbitrary number of generations. It can be readily verified that any Hermitian $2 \times 2$ matrix automatically satisfies Eqs. (25) and one obtains the wellknown result that for two generations and one Higgs doublet the Lagrangian of the SM does not violate CP. For three generations, one can show that there is only one independent condition, corresponding to $p=q=1$. This results from the fact that for $n=3$, the traces involving higher powers are proportional to the trace corresponding to $p=q=1$. One has, for example:

$$
\operatorname{tr}\left[G_{u}^{2}, G_{d}\right]^{3}=\frac{1}{3}\left[\left(\operatorname{tr} G_{u}\right)^{3}-\operatorname{tr}\left(G_{u}\right)^{3}\right] \operatorname{tr}\left[G_{u}, G_{d}\right]^{3}
$$

It can be easily shown [10] that for three generations the condition

$$
\operatorname{tr}\left[G_{u}, G_{d}\right]^{3}=0
$$

is not only a necessary but also a sufficient condition to guarantee CP invariance of the Lagrangian. This is readily shown by explicitly constructing the matrix $W_{L}$ which satisfies Eqs. (22), (23). Note that all our discussion was done prior to gauge symmetry breaking. After $S U(2) \times U(1)$ breaking, quark masses are generated and the conditions of Eq. (25) can be readily written in terms of quark masses, and one obtains, for example:

$$
\operatorname{tr}\left[H_{u}, H_{d}\right]^{3}=0
$$

where $H_{u, d}=M_{u, d} M_{u, d}^{\dagger}$. It is instructive to write the above invariant in terms of quark masses and mixings:

$$
\operatorname{tr}\left[H_{u}, H_{d}\right]^{3}=6\left(\Delta_{21} \Delta_{31} \Delta_{32}\right) \operatorname{Im}\left(V_{11} V_{22} V_{12}^{*} V_{21}^{*}\right)
$$

where $\Delta_{21}=\left(m_{s}^{2}-m_{d}^{2}\right)\left(m_{c}^{2}-m_{u}^{2}\right)$, with analogous expressions for $\Delta_{31}, \Delta_{32}$. This result was to be expected, since we have seen in the previous analysis that $\delta_{\mathrm{KM}}$ can be identified with the strength of CP violation. The quark mass factors were also to be expected, since mass degeneracy in either the up or the down quark sector immediately implies that for three generations all phases can be removed from the CKM matrix and therefore no CP violation arises.

At this stage, it is worth commenting on how the method we have presented here can be extended to theories beyond the SM. As an example, we will take the left-right (LR) symmetric model [11] whose relevant part of the Lagrangian can be written:

$$
\mathcal{L}=\mathcal{L}_{Y}+\mathcal{L}_{W_{L}}+\mathcal{L}_{W_{R}}
$$


where $\mathcal{L}_{W_{L, R}}$ stand for the left-handed $(\mathrm{LH})$ and right-handed $(\mathrm{RH})$ charged-current interactions. The most general CP transformation allowed by the gauge interactions is

$$
\begin{array}{ll}
u_{L} \rightarrow W_{L} C u_{L}^{*} & ; \quad u_{R} \rightarrow W_{R} C u_{R}^{*} \\
d_{L} \rightarrow W_{L} C u_{L}^{*} & ; \quad u_{R} \rightarrow W_{R} C u_{R}^{*}
\end{array}
$$

The important point is that due to the presence of both $\mathrm{LH}$ and $\mathrm{RH}$ charged currents, each of the pairs of chiral fields $\left(u_{L}, d_{L}\right),\left(u_{R}, d_{R}\right)$ are required to transform in the same way under CP. In order for the mass terms arising from $\mathcal{L}_{Y}$ to satisfy the conditions of Eq. (31), they have to satisfy the following equations [12]:

$$
\begin{aligned}
& W_{L}^{\dagger} M_{u} W_{R}=M_{u}^{*} \\
& W_{L}^{\dagger} M_{d} W_{R}=M_{d}^{*}
\end{aligned}
$$

From Eqs. (32), one obtains:

$$
W_{L}^{\dagger} M_{u} M_{d}^{\dagger} W_{L}=\left(M_{d} M_{u}^{\dagger}\right)^{T}
$$

which leads to the condition:

$$
\operatorname{tr}\left[\left(M_{u} M_{d}^{\dagger}\right)^{p}\right]=\operatorname{tr}\left[\left(M_{d} M_{u}^{\dagger}\right)^{p}\right]
$$

The conditions of Eq. (33) are necessary conditions for CP invariance in the LR models, written in terms of invariants. One can deduce from Eqs. (32) other necessary conditions for $\mathrm{CP}$ invariance in the LR models and for two and three generations it is possible to derive a set of invariant conditions which are necessary and sufficient for CP invariance in LR models [12].

\section{Beyond the Standard Model}

\subsection{Motivation}

The success of the SM is truly impressive and therefore one has to carefully justify the motivation for considering extensions of the SM. Actually, the phenomenon of $\mathrm{CP}$ violation may provide some motivation to go beyond the SM. Some of these motivations can be:

(i) - Spontaneous T, CP Violation - One may find it more appealing, as first suggested by T.D. Lee [13], that the Lagrangian be CP invariant and CP be only broken by the vacuum, in analogy with gauge symmetry breaking.

(ii) - Baryogenesis - It is likely [5] that the amount of CP violation present in the SM is not sufficient to produce adequate baryon asymmetry and the electroweak phase transition.

There are some excellent recent reviews [5] on (ii) and therefore we will concentrate here on (i). 


\subsection{Spontaneous $T$, CP violation}

We will address the following questions:

(i) What conditions should a set of vacua satisfy in order to violate $T$, CP?

(ii) What are the minimal extensions of the SM which can lead to spontaneous $T$, CP violation?

\subsubsection{Condition for spontaneous $T$, CP violation}

For definiteness, we will assume the $S U(2) \times U(1)$ model with an arbitrary number of Higgs doublets $\phi_{i}$. However, the condition we will derive can be readily extended for models with other representations of Higgs fields. Since we wish our analysis to be applicable to Lagrangians which may be also invariant under linear transformations which mix the various $\phi_{i}$, we will consider the most general $T$ transformation which leaves the Lagrangian invariant:

$$
T \phi_{i} T^{-1}=U_{i j} \phi_{j}
$$

where $U$ is a unitary matrix which mixes the fields $\phi_{i}$. From Eq. (34) and taking into account the anti-unitarity of the $T$ operator, it follows that if the vacuum is $T$ invariant, i.e.,

$$
T \mid 0>=0
$$

then the following condition holds [14]:

$$
U_{i j}^{*}<0\left|\phi_{j}\right| 0>^{*}=<0\left|\phi_{i}\right| 0>
$$

The simplest way to find whether a given set of vacuum expectation values correspond to a $T$, $\mathrm{CP}$ breaking vacuum, consists of verifying whether there is a unitary matrix $U$ satisfying Eqs. (35), (36). There will be spontaneous $T, C P$ violation if and only if no such unitary matrix exists.

\subsubsection{Minimal extensions of the Standard Model}

We will analyze here the minimal extensions of the SM based on $S U(2) \times U(1)$ which can lead to spontaneous CP violation. It is convenient to consider separately the cases where no non-standard quarks are introduced and where new quarks are added.

Only Standard Quarks - In the framework of an $S U(2) \times U(1)$ gauge theory, the minimal Higgs structure which can lead to spontaneous CP violation capable of explaining the observed CP violation in the kaon sector consists of two Higgs doublets [13]. The most general Higgs potential can be written:

$$
V\left(\phi_{1}, \phi_{2}\right)=V_{0}+\left[\lambda_{1}\left(\phi_{1}^{\dagger} \phi_{2}\right)\left(\phi_{1}^{\dagger} \phi_{2}\right)+\lambda_{2}\left(\phi_{1}^{\dagger} \phi_{2}\right)\left(\phi_{1}^{\dagger} \phi_{1}\right)+\lambda_{3}\left(\phi_{2}^{\dagger} \phi_{2}\right)\left(\phi_{1}^{\dagger} \phi_{2}\right)+\text { h.c. }\right]
$$


where $V_{0}$ denotes the part of the Higgs potential without any phase dependence. We will assume that the Lagrangian is $T$, CP invariant and in this case we may choose, without loss of generality, the coupling constants in $V$ real. It has been shown [13] that for an appropriate finite range of the parameters of the scalar potential, the absolute minimum of the potential is at:

$$
\cos \theta=-\left(4 \lambda_{1} v_{1} v_{2}\right)^{-1}\left[\lambda_{2} v_{1}^{2}+\lambda_{3} v_{2}^{2}\right]
$$

where

$$
<0\left|\phi_{j}^{0}\right| 0>=v_{j} \exp \left(i \theta_{j}\right)
$$

and $\theta=\theta_{2}-\theta_{1}$. In general, the vacuum corresponding to Eq. (39) leads to spontaneous $T$, $\mathrm{CP}$ violation. This can be easily seen by noting that for arbitrary $\lambda_{i}$, the Lagrangian is not invariant under transformations which $\operatorname{mix} \phi_{1}, \phi_{2}$ and as a result the scalar fields transform under $T$ as:

$$
T \phi_{i} T^{-1}=e^{i \alpha_{i}} \phi_{i}
$$

The terms with coefficients $\lambda_{2}, \lambda_{3}$ lead to the constraint $\alpha_{1}=\alpha_{2} \equiv \alpha$. It is then clear that for generic values of $\theta$, one cannot find a value of $\alpha$ such that Eq. (36) is satisfied for both $\phi_{1}, \phi_{2}$, which proves that $T$ is spontaneously violated. The two-Higgs model was suggested at the time when only two fermion generations were known. In this case, CP violation is solely due to Higgs exchange, the dominant contribution to $k^{0}-\bar{k}^{0}$ mixing arising through tree diagrams mediated by neutral Higgs coupled to strangeness-changing scalar currents. For three-fermion generations, it can be verified that the phase $\theta$ induces a KM phase in the quark CKM matrix. This is due to the fact that although there is only one phase $\theta$, there are two completely independent matrices of Yukawa couplings, corresponding to each one of the $\phi_{i}$. The simplest way to recover natural flavour conservation (NFC) in the Higgs sector consists of introducing a $Z_{2}$ symmetry, under which

$$
\phi_{2} \rightarrow-\phi_{2} ; \quad d_{j R} \rightarrow-d_{j R}
$$

with all the remaining fields transforming trivially under $Z_{2}$. The important effect of the $Z_{2}$ symmetry is to forbid couplings of the type $\left(\phi_{1}^{\dagger} \phi_{2}\right)\left(\phi_{i}^{\dagger} \phi_{i}\right)$. One can then show that all the minimae of the new potential are $T$, CP conserving. This is shown by noting that for an appropriate finite range of the parameters, the minimum of the scalar potential is at

$$
<\phi_{1}>=v_{1} ; \quad<\phi_{2}>=v_{2} \exp (i \pi / 2)
$$

Naively one could think that this vacuum violates CP. However, this is not the case [15] since a matrix $U$ exists, satisfying Eqs. (35), (36). Namely:

$$
T \quad\left[\begin{array}{l}
\phi_{1} \\
\phi_{2}
\end{array}\right] \quad T^{-1}=U\left[\begin{array}{l}
\phi_{1} \\
\phi_{2}
\end{array}\right]
$$

with $U=\left[\begin{array}{cc}1 & 0 \\ 0 & -1\end{array}\right]$. If one allows for soft symmetry breaking of the $Z_{2}$ symmetry through terms like $\phi_{1}^{\dagger} \phi_{2}$, one can then obtain [16] spontaneous CP violation. 
The simplest possibility to achieve spontaneous CP violation and keep NFC in the Higgs sector consists of introducing a third Higgs doublet which does not couple to quarks [17]. In this case, it has been shown [15] that for an appropriate range of the parameters of the Higgs potential, one can obtain spontaneous CP violation. Furthermore, it has been pointed out [18] that in this case the CP-violating CKM phase does not generate a KM phase in the quark mixing matrix, and as a result, $\mathrm{CP}$ violation arises exclusively through Higgs exchange.

Extending the fermion sector

We have seen that in the $S U(2) \times U(1)$ gauge theory with only standard families, two Higgs doublets is the minimal structure required to generate spontaneous $\mathrm{CP}$ breaking, capable of accounting for the observed CP violation. We will see next that if one introduces isosinglet quarks one is able to generate spontaneous CP violation [19] with only one Higgs doublet and a Higgs singlet. For definiteness, we will add to the SM only one isosinglet $Q=1 / 3$ quark $D$ and a singlet Higgs $S$. The field content of the model is:

$$
(u d)_{L}^{i} u_{R}^{i}, \quad d_{R}^{i}, D_{R}, D_{L}, \Phi, S
$$

where $i$ is a family index. We will impose CP invariance in the Lagrangian, together with an additional $Z_{2}$ symmetry under which all the fields of the SM transform trivially, while the new fields $D_{L}, D_{R}, S$ are odd. This symmetry is not required in order to have spontaneous CP violation, but it is crucial in order for the model to provide a possible solution [19] of the strong $\mathrm{CP}$ problem [20]. The most general renormalizable $S U(2) \times U(1) \times Z_{2}$ invariant potential can be written as:

$$
\begin{aligned}
V & =V_{\phi}+V_{s}+V_{\phi, s} \\
V_{\phi} & =m^{2} \phi^{\dagger} \phi+\lambda\left(\phi^{\dagger} \phi\right)^{2} \\
V_{s} & =S^{*} S\left[a_{1}+b_{1} S^{*} S\right]+\left[S^{2}+S^{* 2}\right]\left(a_{2}+b_{2} S^{*} S\right)+b_{3}\left(S^{4}+S^{* 4}\right) \\
V_{\phi, S} & =\phi^{\dagger} \phi\left[c_{1}\left(S^{2}+S^{* 2}\right)+c_{2} S^{*} S\right]
\end{aligned}
$$

It can be readily shown that for a range of the parameters of the Higgs potential, the minimum is at:

$$
<\phi^{0}>=\frac{v}{\sqrt{2}} ;<S>=\frac{1}{\sqrt{2}} V e^{i \alpha}
$$

which leads to spontaneous $\mathrm{CP}$ violation. The $S U(2) \times U(1) \times Z_{2}$ invariant Yukawa couplings are:

$$
\begin{aligned}
\mathcal{L}_{Y}= & -\sqrt{2}(\bar{u} \bar{d})_{L}^{i}\left[g_{i j} \phi d_{R}^{j}+h_{i j} \tilde{\phi} u_{R}^{j}\right]-\mu \bar{D}_{L} D_{R}- \\
& -\sqrt{2}\left(f_{i} S+f_{i}^{\prime} S^{*}\right) \bar{D}_{L} d_{R}^{i}+h . c .
\end{aligned}
$$

where all interaction couplings are real, due to $\mathrm{CP}$ invariance. It is convenient to work in the weak basis where the up-quark mass matrix is diagonal. The down-quark mass matrix can be written as:

$$
\mathcal{M}_{d}=\left[\begin{array}{ll}
m_{d} & 0 \\
M_{D} & \mu
\end{array}\right]
$$


where $\left(m_{d}\right)_{i j}=g_{i j} v$ and $\left(M_{D}\right)_{i}=\left[f_{i} V e^{i \alpha}+f_{i}^{\prime} V e^{-i \alpha}\right]$. The matrix $\mathcal{M}_{d}$ is diagonalized by the bi-unitary transformation:

$$
\mathcal{U}_{L}^{\dagger} \mathcal{M}_{d} \mathcal{U}_{R}=\left[\begin{array}{cc}
\bar{m} & 0 \\
0 & \bar{M}
\end{array}\right]
$$

where $\bar{m}=\operatorname{diag}\left(m_{d}, m_{s}, m_{b}\right)$ and $\bar{M}$ is the mass of the heavy quark. It is convenient to write $U_{L}$ in block form:

$$
\mathcal{U}_{L}=\left(\begin{array}{cc}
K & R \\
S & T
\end{array}\right)
$$

where $K$ is the usual $3 \times 3 \mathrm{KM}$ matrix connecting the standard quarks. It can be seen that in the limit $\bar{m}_{i}^{2} / \bar{M}^{2} \ll 1$, one obtains [19]:

$$
K \bar{m}^{2} K^{-1} \cong m_{0}^{2}
$$

where

$$
m_{0}^{2}=m_{d} m_{d}^{\dagger}-\frac{m_{d} M_{D}^{\dagger} M_{D} m_{d}^{\dagger}}{M^{2}}
$$

with $M^{2}=\left(M_{D} M_{D}^{\dagger}+\mu^{2}\right) \cong \bar{M}^{2}$. It can be readily verified that the CKM matrix will have a non-vanishing KM phase even in the limit of very large $V$. The rôle of the isosinglet quark $D$ is clear: through its mixing with standard quarks, it enables CP violation generated by $\langle S\rangle$ to also appear, and unsuppressed, in the light quark mixing.

It should be emphasized that this model, besides providing a solution [19] to the strong $\mathrm{CP}$ problem [20], suggests the exciting possibility of having the observed $\mathrm{CP}$ violation at low energies as the result of physics at a higher energy scale.

\section{Conclusions}

We have reviewed how CP violation arises in electroweak gauge theories. At the Lagrangian level, CP breaking appears when there are at least two sets of couplings which require incompatible CP transformation properties of the fields. We have shown how this can be used to derive weak-basis invariant conditions for having CP invariance in the SM and its extensions. Alternatively, CP may be an exact symmetry of the Lagrangian, spontaneously broken by the vacuum. We have given the conditions which have to be satisfied in order for a given vacuum to violate $T, \mathrm{CP}$, and described the minimal extensions of the SM which can lead to spontaneous CP violation.

We have not covered at all the important topic of how to test experimentally the KM mechanism and how to look for physics beyond the SM through CP violating processes. These topics have been extensively covered in the literature [1], [21]. Here we would like to remark only that the study of heavy flavour physics, and especially $B$-physics, at either $e^{+} e^{-} B$ factories or at hadron colliders, provides an excellent opportunity to test some of the least known aspects of the SM, namely those having to do with Yukawa couplings, the breaking of gauge symmetry and the closely related phenomenon of $\mathrm{CP}$ violation. 


\section{References}

[1] For recent reviews, see:

L. Wolfenstein, Ann.Rev.Nucl.Part.Sci. 36 (1986);

W. Grimus, Fortsch.Phys. 36 (1988) 201;

B. Winstein and L. Wolfenstein, EFI 92-55, to appear in Rev.Mod.Phys. ;

J.F. Donoghue, B.R. Holstein and G. Valencia, Int.J.Mod.Phys. A2 (1987) 319;

Y. Nir and H. Quinn, SLAC.PUB.5737 (1992).

[2] M. Kobayashi and T. Maskawa, Prog.Theor.Phys. 49 (1973) 652.

[3] L. Wolfenstein, Phys.Rev.Lett. 13 (1964) 562.

[4] J.H. Christenson, J.W. Cronin, W.L. Fitch and R. Turlay, Phys.Rev.Lett. 13 (1964) 138.

[5] M.B. Gavela, P. Hernandez, J. Orloff and O. Pène, CERN Preprint TH. 7081/93 (1993);

G.R. Farrar and M.E. Shaposhnikov, CERN Preprint TH. 6734/93 (1993);

A.G. Cohen, D.B. Kaplan and A.E. Nelson, Ann.Rev.Nucl.Part.Sci. 43 (1993).

[6] G.C. Branco and L. Lavoura, Phys.Lett. B208 (1988) 123.

[7] See Particle Data Group, Review of Particle Properties, Phys.Rev. D45 (1992), part 2 and references therein.

[8] L. Wolfenstein, Phys.Rev.Lett. 51 (1983) 1945.

[9] J.D. Bjorken and I. Dunietz, Phys.Rev. D36 (1987) 2109.

[10] J. Bernabéu, G.C. Branco and M. Gronau, Phys.Lett. 169B (1986) 243.

[11] J.C. Pati and A. Salam, Phys.Rev. D10 (1974) 275;

R.N. Mohapatra and J.C. Pati, Phys.Rev. D11 (1975) 2558;

G. Senjanović and R.N. Mohapatra, Phys.Rev. D12 (1975) 1502.

[12] G.C. Branco and M.N. Rebelo, Phys.Lett. B173 (1986) 313.

[13] T.D. Lee, Physics Reports 9C (1974) 143.

[14] G.C. Branco, J.M. Gérard and W. Grimus, Phys.Lett. 136B (1984) 383.

[15] G.C. Branco, Phys.Rev. D22 (1980) 2901.

[16] G.C. Branco and M.N. Rebelo, Phys.Lett. 160B (1985) 117.

[17] S. Weinberg, Phys.Rev.Lett. 37 (1976) 657.

[18] G.C. Branco, Phys.Rev.Lett. 44 (1980) 504.

[19] L. Bento, G.C. Branco and P.A. Parada, Phys.Lett. B267 (1991) 95. 
[20] A. Nelson, Phys.Lett. 136B (1984) 387;

S.M. Barr, Phys.Rev.Lett. 53 (1984) 329.

For excellent reviews on the strong CP problem, see:

R.D. Peccei, DESY Report 88-109 (1988), in CP violation (World Scientific, Singapore);

J. Kim, Physics Reports 150 (1987) 1;

H.Y. Cheng, Physics Reports 158 (1988) 1.

[21] ECFA Workshop on a European B-Meson Factory, ECFA 93/151, Eds. R. Alexan and A. Ali. 